\title{
STRONG DIFFERENTIABILITY PROPERTIES OF BESSEL POTENTIALS
}

BY

DANIEL J. DEIGNAN AND WILLIAM P. ZIEMER(1)

\begin{abstract}
This paper is concerned with the "strong" $L_{p}$ differentiability properties of Bessel potentials of order $\alpha>0$ of $L_{p}$ functions. Thus, for such a function $f$, we investigate the size (in the sense of an appropriate capacity) of the set of points $x$ for which there is a polynomial $P_{x}(y)$ of degree $k<\alpha$ such that
\end{abstract}

$$
\underset{\operatorname{diam}(S) \rightarrow 0}{\lim \sup }(\operatorname{diam} S)^{-k}\left\{|S|^{-1} \int\left|f(y)-P_{x}(y)\right|^{p} d y\right\}^{1 / p}=0
$$

where, for example, $S$ is allowed to run through the family of all oriented rectangles containing the origin.

1. Introduction. Let $\mathscr{F}$ be a family of measurable sets in $\mathbf{R}^{n}$ which satisfy the following properties:

(i) $m(S)>0$ for $S \in \mathscr{F}$,

(1) (ii) for every $\varepsilon>0$, there is a set $S \in \mathcal{F}$ such that $S \subset B(0, \varepsilon)$,

(iii) $\cap\{\bar{S}: S \in \mathcal{F}\}=\{0\}$.

Here $m$ denotes Lebesgue measure and $B(x, r)$ denotes the open $n$-ball centered at $x$ with radius $r$. In this paper we shall investigate the "strong" $L_{p}$ differentiability properties of a certain class of functions with respect to such

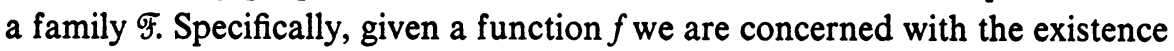
of a polynomial $P_{x}(y)$ of degree $k$ such that

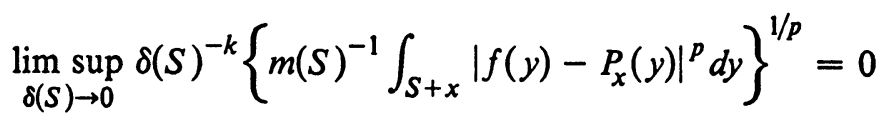

where it is understood that $S \in \mathcal{F}$. The diameter of a set is denoted by $\delta(S)$ and $S+x=\{y+x: y \in S\}$. Whenever (2) is satisfied we shall say that

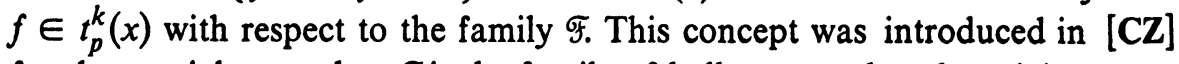
for the special case when $\mathscr{F}$ is the family of balls centered at the origin.

Received by the editors July 14, 1975.

AMS (MOS) subject classifications (1970). Primary 26A54; Secondary 28A10.

(1) Research partially supported by a grant from the National Science Foundation. 
We were led to the problem of strong $L_{p}$ differentiability by certain questions that arose in the study of boundary regularity of solutions of parabolic differential equations [D]. In that study the family $\mathscr{F}$ under consideration was the class of parabolic rectangles of the form $B(0, r) \times\left(0, r^{2}\right)$. This family is "irregular" in the sense that for every $\varepsilon>0$, there is a set $S \in \mathscr{F}$ with $m(s) \cdot m[B(0, \delta(S))]^{-1}<\varepsilon$. As in [D], we shall be primarily concerned with the size of the set where (2) fails to hold.

We will consider the question of strong $L_{p}$ differentiability of functions in the space of Bessel potentials $\mathcal{L}_{p}^{\alpha}=\left\{G_{\alpha} * g: g \in L_{p}\left(\mathbf{R}^{n}\right)\right\}$. Here $G_{\alpha}$ denotes the Bessel kernel of order $\alpha>0$ and $*$ denotes the usual convolution operation. $\mathcal{L}_{p}^{\alpha}$ is a Banach space under the norm $\left\|G_{\alpha} * f\right\|_{\mathbb{L}_{p}^{\alpha}}=\|g\|_{L_{p}}$. When $p>1$ and $\alpha$ is a positive integer, $\mathfrak{L}_{p}^{\alpha}$ is isometric to the Sobolev space $W_{p}^{\alpha}$. For details see [ST].

If $\mathscr{F}=\mathscr{B}$, the family of balls centered at the origin, then the classical theorem of Lebesgue can be rephrased to say that $f \in t_{1}^{0}(x)$ for almost every $x$, whenever $f$ is locally integrable on $\mathbf{R}^{n}$. For this same family $\mathfrak{G}$, higher order differentiability was first considered by Calderón and Zygmund in [CZ], where they prove that if $f \in \mathcal{L}_{p}^{k}$ for integer $k$, then $f \in t_{p}^{k}(x)$ for almost every $x \in \mathbf{R}^{n}$. This result has recently been improved by considering $f \in \mathcal{L}_{p}^{\alpha}$ for arbitrary $\alpha$ and by using an appropriate capacity (or Hausdorff measure) to measure the size of the exceptional sets (cf. [FZ], [BZ], [M2]). All of these results continue to hold if $\mathscr{B}$ is replaced by any family $\mathscr{F}$ that is "regular".

One of the first results to deal with an "irregular" family of sets is due to Zygmund [Z1]. In that paper he considers $\mathscr{F}=\mathscr{R}$, the family of all oriented rectangles which contain the origin. He proves that $f \in t_{1}^{0}(x)$ with respect to $\Re$ for almost every $x$ provided that $f \in L_{p}\left(\mathbf{R}^{n}\right)$ for some $p>1$. This assumption can be weakened slightly by requiring only that

$$
\int_{\mathbf{R}^{n}}|f|\left(\log ^{+}|f|\right)^{n-1}
$$

be finite. See [JMZ] or [ $\mathbf{Z 2}$ ] for a complete discussion. However, some integrability requirement is necessary, for Saks $[\mathbf{S}]$ has provided an example of a function $f \in L_{1}$ such that $f \notin t_{1}^{0}(x)$ for each $x \in \mathbf{R}^{n}$.

A final result worth mentioning is due to Riviere $[\mathbf{R}]$. He considers a Vitali family $\widetilde{V}=\left\{U_{\alpha}\right\}_{\alpha>0}$ of bounded open sets with the following properties:

1. $\alpha<\beta \Rightarrow U_{\alpha} \subset U_{\beta}$ (nestedness),

2. $\cap_{\alpha>0} \overline{\mathrm{U}}_{\alpha}=\{0\}$,

3. $m\left(U_{\alpha}-U_{\alpha}\right) \leqslant A m\left(U_{\alpha}\right)$ for some constant $A$, independent of $\alpha$,

4. $m\left(U_{\alpha}\right)$ is left continuous as a function of $\alpha$.

Here $U_{\alpha}-U_{\alpha}=\left\{x-y: x, y \in U_{\alpha}\right\}$. He then proves that for any locally integrable function $f, f \in t_{1}^{0}(x)$ with respect to $\mathscr{V}$ for almost every $x \in \mathbf{R}^{n}$.

In this paper we shall first generalize the results of Zygmund and Riviere to 
the function classes $\mathcal{L}_{p}^{\alpha}$, and prove that the exceptional set is null with respect to an appropriate capacity. We then consider the question of higher order differentiability. Theorem 2 will show, for example, that the results of [BZ] and [M2] will hold for the family $\Re$ as well as $\mathscr{B}$, provided we assume that $p>1$.

2. First order differentiability. The Bessel potential space $\ell_{p}^{\alpha}$ was defined above. The Bessel capacity associated with this function space is defined as follows. For any set $E \subset \mathbf{R}^{n}$, define

$$
B_{\alpha, p}(E)=\operatorname{Inf}\left\{\|g\|_{L_{p}}^{p}: g \in L_{p}^{+}, G_{\alpha} * g \geqslant 1 \text { on } E\right\} .
$$

This set function is an outer measure on $\mathbf{R}^{n}$ and moreover $B_{\alpha, p}(E)$ $=\operatorname{Inf}\left\{B_{\alpha, p}(G): G \supset E\right.$ and $G$ is open $\}$. If $\alpha=0$ we set $\mathcal{L}_{p}^{0}=L_{p}$ and $B_{0, p}$ $=m$. If $p>1$ and $\alpha p<n$, then $B_{\alpha, p}$ is related to Hausdorff measure of dimension $n-\alpha p$ by the following relations:

1. $H^{n-\alpha p}(E)=0 \Rightarrow B_{\alpha, p}(E)=0$,

2. $B_{\alpha, p}(E)=0 \Rightarrow H^{n-\alpha p+\varepsilon}(E)=0$ for any $\varepsilon>0$.

See Meyers [M1] for further details.

For any family $\mathscr{F}$ defined as in $\S 1$, we introduce the maximal operator

$$
M_{\mathscr{F}} f(x)=\operatorname{Sup}\left\{\frac{1}{M(S)} \int_{S+x} f(y) d y: S \in \mathscr{F}\right\}
$$

defined for every $f \in L_{1}\left(\mathbf{R}^{n}\right)$. Often $\mathcal{F}$ may be chosen to contain only bounded sets, in which case $f$ need be only locally integrable. To prove that functions from a Lebesgue class $L_{p}\left(\mathbf{R}^{n}\right)$ are in $t_{p}^{0}(x)$ almost everywhere with respect to $\mathscr{F}$, it suffices to prove a weak type $(1,1)$ estimate on the maximal operator, i.e.

$$
m\left\{M_{\mathscr{F}} f(x)>t\right\} \leqslant C\|f\|_{L_{1}} / t
$$

for some constant $C$ independent of $f \in L_{1}\left(\mathbf{R}^{n}\right)$. Given (3), we choose functions $f_{n} \in C^{\infty}\left(\mathbf{R}^{n}\right)$ such that $\left\|f-f_{n}\right\|_{L_{p}} \rightarrow 0$ and observe that

$$
\begin{aligned}
\frac{1}{m(S)} \int_{S+x}|f(y)-f(x)|^{p} d y & \leqslant \frac{C_{p}}{m(S)} \int_{S+x}\left|f(y)-f_{n}(y)\right|^{p} d y \\
& +\frac{C_{p}}{m(S)} \int_{S+x}\left|f_{n}(y)-f_{n}(x)\right|^{p} d y+C_{p}\left|f_{n}(x)-f(x)\right|^{p} .
\end{aligned}
$$

The first term on the right is dominated by $C_{p} M_{\mathscr{F}}\left(\left|f-f_{n}\right|^{p}\right)$, and since $f_{n}$ is continuous we have

$$
\overline{\operatorname{Lim}}_{\delta(S) \rightarrow 0} \frac{1}{m(S)} \int_{S+x}|f(y)-f(x)|^{p} d y \leqslant C_{p} M_{\mathscr{F}}\left(\left|f-f_{n}\right|^{p}\right)+C_{p}\left|f_{n}(x)-f(x)\right|^{p} .
$$

In view of (3) and the fact that $f_{n} \rightarrow f$ in measure, we can choose $n$ sufficiently 
large so that the right side is less than $\varepsilon$ except on a set of measure less then $\varepsilon$, hence the left side is zero almost everywhere.

For functions in $\mathscr{L}_{p}^{\alpha}$ we will prove that in fact the same conclusion holds $B_{\alpha, p}$ almost everywhere, by replacing estimate (3) by a capacitary weak type inequality. This can be done provided the maximal operator is a bounded operator on $L_{p}$ (see Adams [A]). The following result was first proved in [D] for the case $\mathscr{F}=\Re$.

THEOREM 1. Let $\mathcal{F}$ be a family of measurable sets satisfying conditions (1), and suppose $M_{\mathscr{F}}$ is of strong type $(p, p)$. That is,

$$
\left\|M_{\mathcal{G}} f\right\|_{L_{p}} \leqslant C\|f\|_{L_{p}}
$$

for some constant $C$ independent of $f \in L_{p}\left(\mathbf{R}^{n}\right), 1 \leqslant p<\infty$. If $f \in \mathfrak{L}_{p}^{\alpha}, \alpha$ $\geqslant 0$, then $f \in t_{1}^{0}(x)$ with respect to $\mathscr{F}$ for $B_{\alpha, p}$ almost every $x \in \mathbf{R}^{n}$.

Proof. The result for $\mathfrak{E}_{p}^{0}=L_{p}$ is precisely that detailed above, so let $\alpha>0$ and let $f=G_{\alpha} * g \in \mathfrak{L}_{p}^{\alpha}$. We first make use of the strong type estimate (4) to prove the following capacitary weak type estimate

$$
B_{\alpha, p}\left\{M_{\mathscr{G}} f(x)>t\right\} \leqslant\left(C / t^{p}\right)\|f\|_{\mathbb{L}_{p}^{\alpha}}^{p} .
$$

This follows by observing that $M_{\mathscr{F}} f \leqslant G_{\alpha} * M_{\mathscr{g}} g$ and, hence,

$$
\begin{aligned}
B_{\alpha, p}\left\{M_{\mathscr{G}} f>t\right\} & \leqslant B_{\alpha, p}\left\{G_{\alpha} * M_{\mathscr{F}} g>t\right\}=B_{\alpha, p}\left\{G_{\alpha} *\left(M_{\mathscr{F}} g / t\right)>1\right\} \\
& \leqslant t^{-p}\left\|M_{\mathscr{G} g}\right\|_{L_{p}}^{p} \leqslant C t^{-p}\|g\|_{L_{p}}^{p}=C t^{-p}\|f\|_{L_{p}^{q}}^{p}
\end{aligned}
$$

using the definition of capacity and estimate (4). Secondly, we will show that any function $f \in \mathscr{L}_{p}^{\alpha}$ can be approximated by continuous functions $f_{\mathcal{E}} \in \mathfrak{L}_{p}^{\alpha}$ such that

$$
\left\|f_{\varepsilon}-f\right\|_{\mathscr{L}_{p}^{\varepsilon}} \rightarrow 0 \text { as } \varepsilon \rightarrow 0
$$

and also that

$$
B_{\alpha, p}\left\{x:\left|f_{\varepsilon}-f\right|(x)>\delta\right\} \rightarrow 0 \text { as } \varepsilon \rightarrow 0 \text { for every } \delta>0 .
$$

To this end we use a standard mollifier argument. Let $\phi \in C_{c}^{\infty}\left(\mathbf{R}^{n}\right)$ be positive with $\int_{\mathbb{R}^{n}} \phi=1$. Set $\phi_{\varepsilon}(x)=\varepsilon^{-n} \phi(x / \varepsilon)$ and define

$$
f_{\varepsilon}=\phi_{\varepsilon} * f=G_{\alpha} *\left(\phi_{\varepsilon} * g\right) \text {. }
$$

Then

$$
\left\|f_{\varepsilon}-f\right\|_{\mathfrak{L}_{p}^{\alpha}}=\left\|G_{\alpha} *\left(G_{\varepsilon}-G\right)\right\|_{\mathfrak{L}_{p}^{\alpha}}=\left\|g_{\varepsilon}-g\right\|_{L_{p}}
$$


and since $g_{\varepsilon} \rightarrow g$ in $L_{p},(6)$ is proved. Also notice that

$$
\begin{aligned}
B_{\alpha, p}\left\{x:\left|f_{\varepsilon}(x)-f(x)\right|>\delta\right\} & \leqslant B_{\alpha, p}\left\{x: G_{\alpha} *\left|g_{\varepsilon}-g\right|(x)>\delta\right\} \\
& \leqslant \delta^{-p}\left\|g_{\varepsilon}-g\right\|_{L_{p}^{\alpha}}^{p} \rightarrow 0 \text { as } \varepsilon \rightarrow 0
\end{aligned}
$$

so that (7) holds as well. Now fix $\eta>0$ and choose $\varepsilon$ so small that $\left\|f_{\varepsilon}-f\right\|_{\mathbb{L}_{p}^{a}}^{p} \leqslant \eta^{p+1} / C$, where $C$ is the constant of (4), such that

$$
B_{\alpha, p}\left\{x:\left|f_{\varepsilon}-f\right|(x)>\eta\right\}<\eta
$$

Then

$$
\begin{aligned}
\frac{1}{m(S)} \int_{S+x}|f(y)-f(x)| d y \leqslant & \frac{1}{m(S)} \int_{S+x}\left|f(y)-f_{\varepsilon}(y)\right| d y \\
& +\frac{1}{m(S)} \int_{S+x}\left|f_{\varepsilon}(y)-f_{\varepsilon}(x)\right| d y+\left|f_{\varepsilon}(x)-f(x)\right|
\end{aligned}
$$

Note that the last term is $\left\langle\eta\right.$ except on a set of $B_{\alpha, p}$ capacity $<\eta$. The first term is bounded by $M_{\mathscr{F}}\left(\left|f-f_{\varepsilon}\right|\right)$, which, according to (5) is less than $\eta$ except on a set of $B_{\alpha, p}$ capacity less than $\left(C / \eta^{p}\right)\left\|f-f_{\varepsilon}\right\|_{\mathbb{L}_{p}^{\alpha}}^{p}<\eta$. The middle term becomes negligible as $\delta(S) \rightarrow 0$ since $f_{\varepsilon}$ is continuous. Altogether, we conclude

$$
\overline{\operatorname{Lim}}_{\delta(S) \rightarrow 0} \frac{1}{m(S)} \int_{S+x}|f(y)-f(x)| d y<2 \eta
$$

except on a set of $B_{\alpha, p}$ capacity less than $2 \eta$. Since $\eta$ is arbitrary, this proves the theorem. Notice that we have specified the constant polynomial $P_{x}$ to be $f(x)$, which is defined, of course, $B_{\alpha, p}$ almost everywhere. Q.E.D.

Corollary 1. If $f \in \mathcal{L}_{p}^{\alpha}$ with $\alpha>0$ and $1<p<\infty$, then $f \in t_{1}^{0}(x)$ with respect to $\mathscr{F}$ for $B_{\alpha, p}$ almost every $x$ if

1. $\mathscr{F}=\mathscr{B}$, the family of all balls centered at the origin,

2. $\mathcal{F}=\Re$, the family of all rectangles containing the origin,

3. $\mathscr{F}=\mathfrak{T}$, the family defined in $\S 1$.

Proof. In view of Theorem 1, we need only verify hypothesis (4) for each family. For $B$, this result is well known and appears, for example, in Stein [ST]. For $\Re$, estimate (4) is proved in [JMZ].

The proof of (4) for the family $\checkmark$ follows from the weak type $(1,1)$ estimate

$$
m\left\{x: M_{\curlyvee} f(x)>t\right\} \leqslant(C / t)\|f\|_{L_{1}}
$$

for $f \in L_{1}\left(\mathbf{R}^{n}\right)$. This extimate is proved in [R]. To prove (4) from this, we use the familiar argument which appears, for example, on p. 7 of [ST]. Set 


$$
f_{s}(x)= \begin{cases}f(x) & \text { if }|f(x)| \geqslant s / 2, \\ 0 & \text { otherwise }\end{cases}
$$

Then $M_{\Upsilon} f(x) \leqslant M_{\Upsilon} f_{s}(x)+s / 2$ and so

$$
m\left\{x: M_{\Upsilon} f(x)>s\right\} \leqslant m\left\{x: M_{\Upsilon} f_{s}(x)>s / 2\right\} \leqslant(2 C / s)\left\|f_{s}\right\|_{L_{1}}
$$

by (8) since $f_{s} \in L_{1}\left(\mathbf{R}^{n}\right)$. If we set $\lambda(s)=m\left\{x: M_{\Upsilon} f(x)>s\right\}$, then

$$
\begin{aligned}
\int_{\mathbf{R}^{n}}\left|M_{\mho} f\right|^{p} d x=p \int_{0}^{\infty} s^{p-1} \lambda(s) d s \leqslant p \int_{0}^{\infty} 2 C s^{p-2}\left\|f_{s}\right\|_{L_{1}} d s \\
\quad=2 C p \int_{0}^{\infty} s^{p-2} \int_{|f|>s / 2}|f(x)| d x d s=2 C p \int_{\mathbf{R}^{n}}|f(x)| \int_{0}^{2|f(x)|} s^{p-2} d s d x \\
\quad=\frac{2 C p}{p-1} \int_{\mathbf{R}^{n}}|f(x)| 2|f(x)|^{p-1} d x=\frac{4 C p}{p-1}\|f\|_{L_{p}}^{p}
\end{aligned}
$$

which verifies (4) and completes the proof of the corollary.

3. Higher order differentiability. In this section we shall discuss the concept of higher order differentiability with respect to irregular families. Theorem 2 will imply, for example, that $\mathfrak{L}_{p}^{\alpha}$ functions are in $t_{1}^{k}(x)$ with respect to $R$ for $B_{\alpha-k, p}$ almost every $x \in \mathbf{R}^{n}$. Unfortunately, the additional assumption we shall make in Theorem 2 does not apply to arbitrary families $\mathcal{V}$, and the question of higher order differentiability with respect to such a family remains open.

THEOREM 2. Let $\mathscr{F}$ be a family of measurable sets satisfying conditions (1) and suppose in addition that $\sigma_{F}$ is closed under dilations. That is, if $0<t<1, t \cdot S$ $=\{t y: y \in S\}$ is in $\mathscr{F}$ whenever $S$ is. Suppose $M_{\mathscr{f}}$ satisfies condition (4).

If $f \in \mathfrak{L}_{p}^{\alpha}, \alpha \geqslant 1$, and if $k \leqslant \alpha$ is an integer, then $f \in t_{1}^{k}(x)$ with respect to $\mathscr{F}$ for $B_{\alpha-k, p}$ almost every $x \in \mathbf{R}^{n}$.

In order to prove the theorem, we shall make use of the following result, which is an improvement of a similar result proved in [GZ].

LeMma 1. Let $f \in \mathfrak{L}_{p}^{\alpha}$ with $1 \leqslant \alpha<\infty$. Then, for $B_{\alpha, p}$ almost every $x \in \mathbf{R}^{n}$, $f$ is absolutely continuous on $H^{n-1}$ almost every ray emanating from $x$. Moreover, on such a ray,

$$
f(x+z)-f(x)=\int_{0}^{1} \nabla f(x+t z) \cdot z d t
$$

Proof of Lemma 1. Let $f=g_{\alpha} * g \in \mathcal{L}_{p}^{\alpha}$ and let $f_{i}=G_{\alpha} * g_{i}$ be $C^{\infty}$ mollifiers of $f$. Then $\left\|f_{i}-f\right\|_{\mathfrak{L}_{p}^{\alpha}} \rightarrow 0$ as $i \rightarrow \infty$, and there is a subsequence $\left\{f_{j}\right\}$ that converges for $B_{\alpha, p}$ almost every $x$. That is, for some set $E$ with $B_{\alpha, p}(E)=0$, 


$$
f_{j}(x) \rightarrow f(x) \text { for every } x \in \mathbf{R}^{\eta} \backslash E .
$$

For this subsequence, $\nabla f_{j} \rightarrow \nabla f$ in $\mathcal{L}_{p}^{\alpha-1}$ (see [ST, Lemma 3, p. 136]), and therefore

$$
\nabla f_{j}-\nabla f=G_{\alpha-1} * h_{j}
$$

where $h_{j} \rightarrow 0$ in $L_{p}\left(\mathbf{R}^{n}\right)$. But then

$$
G_{1} *\left|\nabla f_{j}-\nabla f\right|(x)=G_{1} *\left|G_{\alpha-1} * h_{j}\right|(x) \leqslant G_{\alpha} *\left|h_{j}\right|(x) .
$$

Since $h_{j} \rightarrow 0$ in $L^{p}\left(\mathbf{R}^{n}\right), G_{\alpha} *\left|h_{j}\right|(x) \rightarrow 0$ for $B_{\alpha, p}$ almost every $x$, hence so does $G_{1} *\left|\nabla f_{j}-\nabla f\right|(x)$ by the above inequality. For any such point $x$ we have

$$
\begin{aligned}
G_{1} *\left|\nabla f_{j}-\nabla f\right|(x) & =\int_{\mathbb{R}^{n}} G_{1}(z)\left|\nabla f_{j}(x-z)-\nabla f(x-z)\right| d z \\
& \geqslant \int_{B(0, R)} G_{1}(z)\left|\nabla f_{j}(x-z)-\nabla f(x-z)\right| d z \\
& \cdot \int_{\partial B(0,1)} \int_{0}^{R} \frac{G_{1}(r y)}{|r y|^{n-1}}\left|\nabla f_{j}(x-r y)-\nabla f(x-r y)\right| d r d H^{n-1}(y)
\end{aligned}
$$

where the last step is integration in polar coordinates. Using the estimate $G_{1}(z) \geqslant C|z|^{n-1}$ for $|z|<R$ (see [ST, p. 132]) we conclude from the above that

$$
\int_{\partial B(0,1)} \int_{0}^{R}\left|\nabla f_{j}(x-r y)-\nabla f(x-r y)\right| d r d H^{n-1}(y) \rightarrow 0
$$

for $B_{\alpha, p}$ almost every $x$. For such a point $x$, we may choose a further subsequence $f_{k}$ such that

$$
\int_{0}^{R}\left|\nabla f_{k}(x-r y)-\nabla f(x-r y)\right| d r \rightarrow 0
$$

for $H^{n-1}$ almost every $y \ni \partial B(0,1)$. Moreover we may assume that $x-r y$ $\notin E$ for any such point $y, 0<r<R$ (see [GZ]). If $z=x-r y$ is any such point, since $f_{k} \in C^{\infty}\left(\mathbf{R}^{n}\right)$ we have

$$
f_{k}(x+z)-f_{k}(x)=\int_{0}^{1} \nabla f_{k}(x+t z) \cdot z d t .
$$

Letting $k \rightarrow \infty$, in view of (9) and (10) we may replace $f_{k}$ by $f$ in the above. Finally, choose a sequence $R_{j} \rightarrow \infty$ to prove the lemma. Q.E.D.

Proof OF ThEOREM 2. Let $f \in \mathfrak{L}_{p}^{\alpha}, \alpha \geqslant 1$, and let $k \leqslant \alpha$ be an integer. Let $P_{x}(y)$ be the Taylor polynomial of degree $k$, i.e. 


$$
P_{x}(y)=\sum_{0<|\beta|<k} \frac{D^{\beta} f(x)}{\beta !}(y-x)^{\beta} .
$$

Here $\beta=\left(\beta_{1}, \ldots, \beta_{n}\right)$ is a multi-index,

$$
|\beta|=\sum_{j=1}^{n} \beta_{j}, \quad D^{\beta}=\frac{\partial^{|\beta|}}{\partial x_{1}^{\beta_{1}} \cdots \partial x_{n}^{\beta_{n}}}, \quad \beta !=\beta_{1} ! \beta_{2} ! \cdots \beta_{n} !,
$$

and $z^{\beta}=z_{1}^{\beta_{1}} z_{2}^{\beta_{2}} \cdots z_{n}^{\beta_{n}}$. Note that if $|\beta| \leqslant k$, then $D^{\beta} f \in \mathcal{L}_{p}^{\alpha-k}$, and since such functions are defined up to sets of $B_{\alpha-k, p}$ capacity zero, $P_{x}(y)$ is defined for $B_{\alpha-k, p}$ almost all $x \in \mathbf{R}^{n}$.

Let $R_{x}(y)=f(y)-P_{x}(y)$ and notice that if $|\gamma|=k$ then

$$
D^{\gamma} R_{x}(y)=D^{\gamma} f(y)-D^{\gamma} f(x) .
$$

Since $D^{\gamma} f \in \mathcal{E}_{p}^{\alpha-k}$, we know from Theorem 1 that

$$
\overline{\operatorname{Lim}}_{\delta(S) \rightarrow 0 ; S \in \mathscr{F}} \frac{1}{m(S)} \int_{S+x}\left|D^{\gamma} R_{x}(y)\right| d y=0
$$

at $B_{\alpha-k, p}$ almost every $x$, whenever $|\gamma|=k$. Now let $\nu$ be a multi-index such that $|\nu|=k-1$. Since $D^{\nu} f \in \mathfrak{L}_{p}^{\alpha-k}$, we know from Lemma 1 that for $B_{\alpha-k, p}$ almost every point $x, D^{y} f$ is absolutely continuous on $H^{n-1}$ almost every ray emanating from $x$. Hence on such a ray, $D^{y} R_{x}(y)$ is also absolutely continuous and

$$
\left|D^{\nu} R_{x}(x+z)-D^{v} R_{x}(x)\right| \leqslant \int_{0}^{1}\left|\nabla\left(D^{\nu} R_{x}\right)(x+t z) \cdot z\right| d t .
$$

Since this estimate holds for almost every $z \in \mathbf{R}^{n}$, we may integrate both sides with respect to $z$ over a set $S \in \mathscr{F}$, yielding

$$
\begin{aligned}
\int_{S}\left|D^{y} R_{x}(x+z)-D^{\nu} R_{x}(x)\right| d z & \leqslant \int_{S} \int_{0}^{1}\left|\nabla\left(D^{y} R_{x}\right)(x+t z) \cdot z\right| d t d z \\
& \leqslant \int_{0}^{1} \int_{S} \delta(S)\left|\nabla\left(D^{y} R_{x}\right)(x+t z)\right| d z d t \\
& =\int_{0}^{1} \frac{\delta(S)}{t^{n}} \int_{t \cdot S}\left|\nabla\left(D^{y} R_{x}\right)(x+z)\right| d z d t
\end{aligned}
$$

The second inequality above is obtained by interchanging the order of integration and using the Schwartz inequality, and the final step is just the change of variables $z \mapsto t z$. We now divide both sides by $m(s) \delta(s)$ and use the transformation $x+z \mapsto z$, noting that $t^{n} m(S)=m(t \cdot S)$. 


$$
\begin{aligned}
\frac{1}{\delta(S)} \frac{1}{m(S)} \int_{S+x} & \left|D^{\nu} R_{x}(z)-D^{\nu} R_{x}(0)\right| d z \\
& \leqslant \int_{0}^{1} \frac{1}{m(t \cdot S)} \int_{t \cdot S+x}\left|\nabla\left(D^{\nu} R_{x}\right)(z)\right| d z d t
\end{aligned}
$$

Since all derivatives of $R_{x}$ of order $k$ satisfy (11) and since for $0<t<1, t \cdot S$ $\in \mathscr{F}$ with $\delta(t \cdot S) \leqslant \delta(S)$, we conclude the existence of a number $\delta$ such that the right side of the above is less than $\varepsilon$ whenever $\delta(S)<\delta$. Since $D^{\nu} R_{x}(0)$ $=0$, this implies

$$
\varlimsup_{\delta(S) \rightarrow 0 ; S \in \mathscr{F}} \frac{1}{\delta(S)} \frac{1}{m(S)} \int_{S+x}\left|D^{\nu} R_{x}(y)\right| d y=0
$$

for $B_{\alpha-k, p}$ almost every $x$, whenever $|\nu|=k-1$. This estimate replaces (11) for derivatives of order $k$. Now let $\eta$ be a multi-index with $|\eta|=k-2$. Arguing just as above we conclude that

$$
\overline{\operatorname{Lim}}_{\delta(S) \rightarrow 0 ; S \in \mathcal{G}} \frac{1}{\delta(S)^{2}} \frac{1}{m(S)} \int_{S+x}\left|D^{\eta} R_{x}(y)\right| d y=0
$$

and inductively that

$$
\overline{\operatorname{Lim}}_{\delta(S) \rightarrow 0 ; S \in \mathcal{F}} \frac{1}{\delta(S)^{k}} \frac{1}{m(S)} \int_{S+x}\left|D^{0} R_{x}(y)\right| d y=0
$$

which proves the theorem. Q.E.D.

COROLlaRy 2. If $\in \mathcal{L}_{p}^{\alpha}$ with $\alpha \geqslant 1,1<p<\infty$, and if $k \leqslant \alpha$ is an integer, then $f \in t_{1}^{k}(x)$ with respect to $\Re$ for $B_{\alpha-k, p}$ almost every $x \in \mathbf{R}^{n}$.

The exponent 1 in Theorems 1 and 2 can be improved if one is willing to accept a larger exceptional set. For example, if $1<q<p$ and $k \leqslant \alpha-1$, then one can show that $f \in t_{q}^{k}(x)$ for $B_{1, s}$ almost every $x$, where $s<p$ satisfies the equation $s p /(p-s)=n p /(n-p)(q-1)$. This follows from the observation that if $|\gamma|=k,\left|D^{\gamma} f\right|^{q} \in W^{1, s}\left(\mathbf{R}^{n}\right)=\mathcal{L}_{s}^{1}$. Applying Theorem 1 to $\left|D^{\gamma} f\right|^{q}$ we obtain estimate (11) with exponent $q$ for $B_{1, s}$ almost every $x$. Making the appropriate changes in the rest of the argument now proves the claim.

The question as to whether the exponent 1 can be improved in the general case without altering the capacity seems to be open. Saks' example [S], seems to indicate that one cannot expect $f$ to lie in $t_{p}^{k}(x)$ even almost everywhere.

Finally, in the definition of strong $L_{p}$ differentiability (see (2)), one may be tempted to replace $\delta(S)^{k}$ by $m(S)^{k / n}$. We conclude by exhibiting an example which shows that $\delta(S)^{k}$ cannot be replaced by $m(S)$ raised to any power.

Let $f(x, y)=x^{2}$ and consider the family $\mathscr{F}=\left\{R_{\varepsilon}\right\}_{\varepsilon}>0$ of rectangles of the form $R_{\varepsilon}=\left\{(x, y):-\varepsilon<x<\varepsilon,-\varepsilon^{3}<y<\varepsilon^{3}\right\}$. After mollification outside a 
larger set, we may assume that $f \in \mathcal{L}_{p}^{\alpha}\left(\mathbf{R}^{2}\right)$ for all $\alpha \geqslant 0, p \geqslant 1$. The Taylor polynomial of degree one at the origin is simply $P_{0}(y) \equiv 0$, and it is easily verified that

$$
\frac{1}{m\left(R_{\varepsilon}\right)^{1 / 2}} \frac{1}{m\left(R_{\varepsilon}\right)} \int_{R_{\varepsilon}}|f(x, y)| d x d y=\frac{1}{\left(4 \varepsilon^{4}\right)^{1 / 2}} \frac{1}{4 \varepsilon^{4}} \frac{4 \varepsilon^{6}}{3}=\frac{1}{6} \nrightarrow 0 .
$$

Similar behavior is exhibited at points other than the origin. Moreover, by modifying the rectangles $R_{\varepsilon}$, one can show it is impossible to replace $\delta(S)^{k}$ by $m(S)$ to any power.

\section{REFERENCES}

[A] David R. Adams, Maximal operators and capacity, Proc. Amer. Math. Soc. 34 (1972), 152-156. MR 50 \#2807.

[BZ] T. Bagby and W. P. Ziemer, Pointwise differentiability and absolute continuity, Trans. Amer. Math. Soc. 191 (1974), 129-148. MR 49 \#9129.

[BF] H. Busemann and W. Feller, Zur differentiation der Lebesgueschen Integrale, Fund. Math. 22 (1934), 226-256.

[CZ] A. P. Calderón and A. Zygmund, Local properties of solutions of elliptic partial differential equations, Studia Math. 20 (1961), 171-225. MR 25 \#310.

[D] Daniel J. Deignan, Boundary regularity of weak solutions to a quasilinear parabolic equation, Doctoral Dissertation, Indiana University, 1974.

[FZ] H. Federer and W. P. Ziemer, The Lebesgue set of a function whose distribution derivatives are p-th power summable, Indiana Univ. Math. J. 22 (1972), 139-158.

[GZ] Ronald Gariepy and W. P. Ziemer, Behavior at the boundary of solutions of quasilinear elliptic equations, Arch Rational Mech. Anal. 56 (1974/75), 372-384. MR 50 \# 7807.

[JMZ] B. Jessen, J. Marcinkiewicz and A. Zygmund, Note on the differentiability of multiple integrals, Fund. Math. 25 (1935), 217-234.

[M1] N. G. Meyers, $A$ theory of capacities for potentials of functions in Lebesgue classes, Math. Scand. 26 (1970), 255-292 (1971). MR 43 \#3474.

[M2] -, Taylor expansion of Bessel potentials, Indiana Univ. Math J. 23 (1973/74), 1043-1049. MR 50 \# 980.

[M] A. P. Morse, Perfect blankets, Trans. Amer. Math. Soc. 61 (1947), 418-442. MR 8, 571.

[R] N. Riviere, Singular integrals and multiplier operators, Ark. Mat. 9 (1971), 243-278.

[S] S. Saks, Remark on the differentiability of the Lebesgue indefinite integral, Fund. Math. 22 (1934), 257-261.

[ST] E. M. Stein, Singular integrals and differentiability properties of functions, Princeton Univ. Press, Princeton, N. J., 1970. MR 44 \# 7280.

[Z1] A. Zygmund, On the differentiability of multiple integrals, Fund. Math. 23 (1934), 143-149.

[Z2] — Trigonometric series, 2nd ed., Cambridge Univ. Press, New York, 1959. MR 21 \#6498.

Department of Mathematics, Indiana University, Bloomington, Indiana 47401 